\title{
Modeling of structuring processes at hardening of expanding cements and concretes on their basis
}

\author{
Igor Kharchenko ${ }^{1, *}$, Alexander Panchenko ${ }^{1}$, Alexey Kharchenko ${ }^{1}$, and Vyacheslav \\ Alekseev ${ }^{1}$ \\ ${ }^{1}$ Moscow State University of Civil Engineering, Yaroslavskoe shosse, 26, Moscow, 129337, Russia
}

\begin{abstract}
This paper reports the results of experimental research of the effect of strain-restriction conditions on the structure and properties of sulfoaluminate expanding cementitious materials. Theoretical analysis of the development of pattern formation processes is performed by applying the developed rheological model, illustrating features of the kinetics of structure-forming processes according to the ratio of the potential index of extension with linear, flat and volumetric limitation of deformations of the extension. The results show that rheological model adequately correlates with the results of experimental studies and can be described mathematically. Found that with the volume limitation of deformations arising when mixing crystallographic phases with high density and strength, the pore structure of the cement stone contains mainly gel pores. This is the main prerequisite for obtaining a dense, high-strength and durable structure of cement stone and concrete on its basis.
\end{abstract}

\section{Introduction}

Provision of operating reliability of concrete and reinforced concrete building structures is to a significant extent defined with quality and durability of concrete used for their production. Guaranteed provision of designed physical and chemical properties of concrete is in turn defined with numerous factors and their mutual influence, which are at the present time sufficiently well-known. Factors defining the quality of concrete first of all include the influence and the properties of mineral binders, inert aggregates, and mutual influence of "external" and "internal" hardening conditions for binders and concretes on their basis. Herewith, "internal" conditions firstly imply for temperature and humidity conditions of hardening, methods of mixing, placing and compacting of concrete mix, methods and conditions of restriction of possible strain accompanying concrete hardening. Among numerous "internal" factors defining the quality of concrete mixes and concrete are chemical and mineralogical and grain-size composition of mineral binder, correlation of components in concrete mix, quantity and quality of additives in the mix [1,2]. In order to provide the designed properties of concrete mix and hardened concrete, as a rule, it is

\footnotetext{
* Corresponding author: iharcenko@mail.ru
} 
necessary to conduct a complex of laboratory studies, which scope minimization is possible on the basis of analysis of structuring processes development using concrete rheological model $[3,4,5,6]$. According to the proposed model the influence of "internal" and "external" factors on structure and properties of hardening cement stone and concrete on its basis demonstrates itself via a piston system „V“, which is connected to a piston vessel " $\mathrm{R}$ ”. (Figure 1).

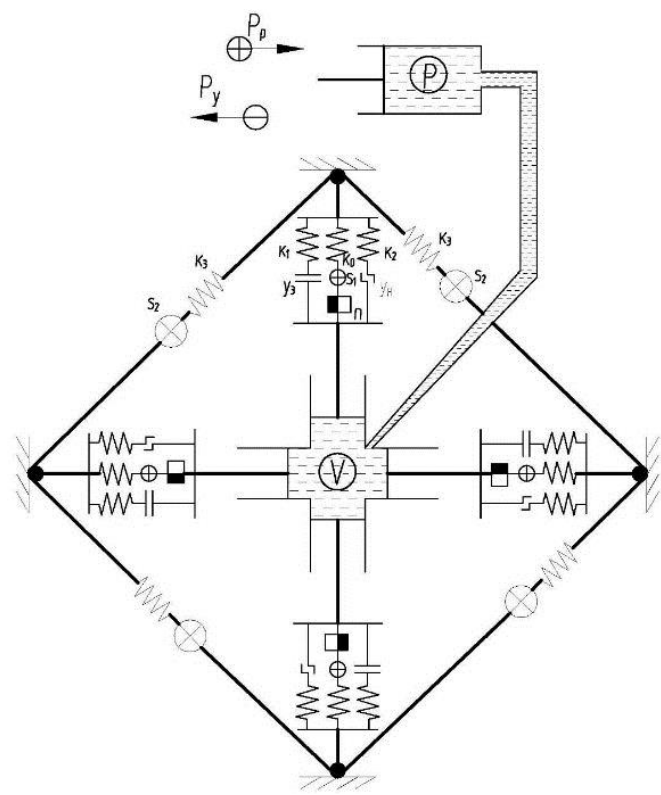

Fig. 1. Rheological model of cement stone structure: $\mathrm{Rp}$ is the positive stress at expansion; Ry is the negative stress at shrinkage; $\mathrm{R}$ is the vessel with incompressible liquid characterizing potential change of cement stone volume: increase of volume at hardening of expanding cements and reduction of volume at their shrinkage; $\mathrm{KO}$ is the spring characterizing structure strengthening at the expense of reduction of total volume of pores and capillaries; K1 is the spring characterizing structure strengthening at closing of contact elements "Uz"; K2 is the spring characterizing structure strengthening at the expense of elimination of defects (micro- and macrocracks) at closing of contacts $\mathrm{Un}$; K3 is the spring characterizing the quality of cement stone macrostructure; $\mathrm{P}$ is the element characterizing plastic properties of cement stone structure and is defined with plastic yield or plastic strain modulus; $\mathrm{S} 1$ is the element characterizing brittleness (crack resistance) of cement stone microstructure; S2 is the element characterizing brittleness (crack resistance) of cement stone macrostructure; $\mathrm{V}$ is the cement stone volume: volume increase at hardening of expanding cement systems; volume reduction at hardening of standard cements at shrinkage strain development; $\mathrm{O}$ is the fixed link restricting the strain.

\section{Experimental investigation}

The entire piston system is a closed hermetically sealed system filled with incompressible liquid. Any piston movement in cylinder " $R$ " causes movement of four pistons in plane system or of six pistons in spatial system, which manifests itself in elements $(\mathrm{KO}, \mathrm{K} 1, \mathrm{~K} 2$, $\mathrm{S} 1, \mathrm{Y} 3, \mathrm{Y} 4)$ characterizing cement stone microstructure and concrete macrostructure (K3, $\mathrm{S} 2$ ). All model elements are connected with rigid links via rotating blocks located in model corners. Piston "R" movement to the right in volume "V" causes excessive pressure, which models the increase of volume of concrete and reinforced concrete structures with the application of composite binders consisting of basic Portland cement with expanding 
additives. At piston "P" movement to the left the volume in piston system «V» reduces, which models the integral volume of shrinkage strain revealing at cement stone hardening.

The entire piston system is a closed hermetically sealed system filled with incompressible liquid. Any piston movement in cylinder " $R$ " causes movement of four pistons in plane system or of six pistons in spatial system, which manifests itself in elements $(\mathrm{KO}, \mathrm{K} 1, \mathrm{~K} 2, \mathrm{~S} 1, \mathrm{Y} 3, \mathrm{Y} 4)$ characterizing cement stone microstructure and concrete macrostructure (K3, S2). All model elements are connected with rigid links via rotating blocks located in model corners. Piston " $\mathrm{R}$ " movement to the right in volume "V" causes excessive pressure, which models the increase of volume of concrete and reinforced concrete structures with the application of composite binders consisting of basic Portland cement with expanding additives. At piston "P" movement to the left the volume in piston system «V» reduces, which models the integral volume of shrinkage strain revealing at cement stone hardening.

\section{Analytical procedure}

As previously noted, qualitative and quantitative condition of microstructure of hardening cement stone and concrete may be described with the elastic spring elements KO, K1 and $\mathrm{K} 2$, which characterize the change of microstructure condition at the expense of reduction and increase in pore volume (KO). Microstructure strengthens at the expense of improvement of contact between the cement stone and the filler (K1), when it switches to operation via contact element «Y3».

Additional strengthening of microstructure, increase of its crack resistance, tensile strength and tensile bending strength reveals via contact element (K2), when contact element «Y4» switches to work. Elements «S1» and «S2» characterize crack resistance of cement stone micro- and macrostructure, respectively. It may numerically be assessed with the value of the tensile strength of cement stone and with the correlation between the tensile strength and compression strength.

Plastic strain of cement stone and concrete on its basis is characterized with element " $\mathrm{P}$ " and may numerically be assessed with plastic yield value for cement stone and concrete, or by plastic strain modulus. Strength characteristics of macrostructure of cement stone or concrete on its basis are described with the spring element «K 3 » and are numerically assessed by the value of compression strength. Elements «O» located in the model corners characterize "external" influence factors that reveal themselves in restriction of expansion deformations (at application of expanding or self-stressing cements), or compression at shrinkage during hardening of cement stone and concrete on its basis. Work of the presented structural model may be illustrated with the example of analysis of processes accompanying hardening of expanding cements. In this regard it is suggested to perform the analysis of model condition in three basic states: complete absence of restriction of external deformations (Figure 2.a); partial restriction of deformations (Figure 2.b); complete restriction of deformations (Figure 2.c)

The influence of external pressure «Rr» on piston in cylinder «R» causes excessive pressure, which is transmitted via four cylinders $« \mathrm{~V} »$ to corresponding pistons. These pistons, in turn, transmit via elements $\langle\mathrm{P} »$ and $« \mathrm{~S} 1 »$ to the spring «Ko» the pressure, under which it compresses, turning on the strengthening elements «Y3» and «Y4». At ongoing process of expansion springs «K1» and «K2» switch to work. In case of absence of strain restrictions and ongoing process of expansion the spring «K3» extends. At further growth of tensile stress in spring «K $3 »$ it is transmitted to element «S2», which upon reaching of critical value it tears apart, which shows up in formation of cracks across the surface of cement stone and concrete on its basis (Figure 3). 


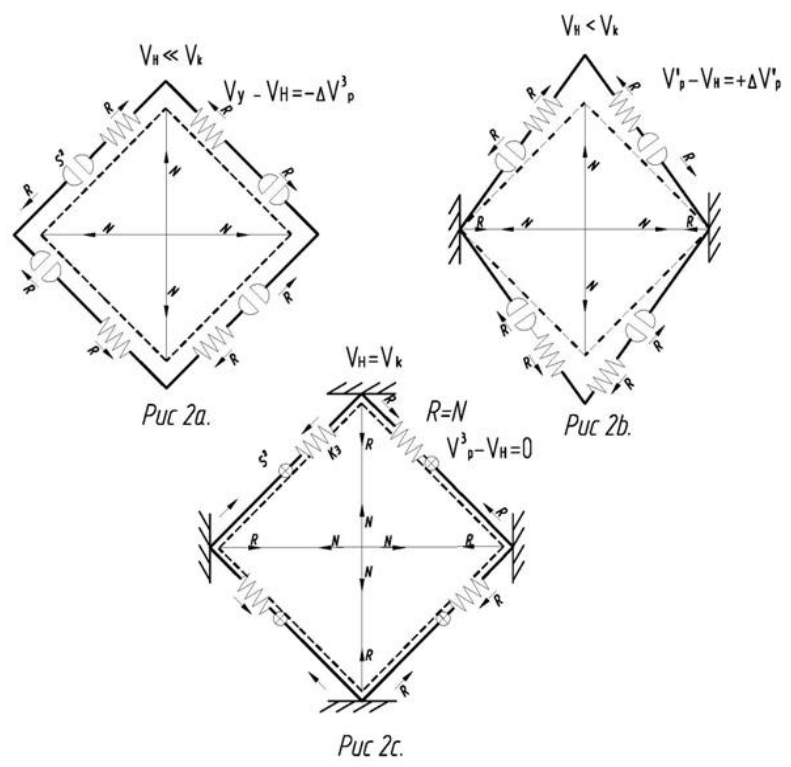

Fig. 2. Changes in rheological model state: expansion without restriction of deformations (2a); expansion at single-axis restriction of deformations (2b); expansion at complete restriction of deformations (2c). Legend: $\mathrm{R}$ is the stress caused with external restrictions of deformations; $\mathrm{N}$ is the internal stress in the cement stone structure; $\mathrm{Vn}$ is the initial volume of cement stone; Vk is the volume of cement stone after appearing of shrinkage or expansion deformations.

\section{Comparison of predictions and experimental results}

At single-axis restriction of expansion deformations (Figure 2b), in the early stage of hardening structuring processes develop according to the above scheme. At switching to work of springs «K1» and «K2» via strengthening elements «Y3» and «Y4» in units restricting deformations the chemical energy of expansion is consumed for compaction and strengthening of pore structure. Strengthening process develops until tensile force «N» does not exceed reactive force «R». At further development of expansion process this equilibrium is disturbed, and the volume increases in the direction perpendicular to the axis of deformation restriction. Increase in volume gives the specimen barrel-like shape (Figure $3)$.

Herewith, the increase in volume at single-axis restriction of deformations does not exceed $25-30 \%$ of the volume that appeared without deformation restriction [3]. At twoaxis restriction of expansion deformations (Figure $2 \mathrm{~b}$ ) released chemical energy is almost completely consumed for compaction of pore structure of cement stone and concrete on its basis.

In model it reveals itself in switching to work of strengthening elements «Y3» and «Y4», and springs Ko, K1 and K2 that only compress. Herewith, element «S1» characterizing system brittleness works only in compression. Elements, charactering macrostructure state, «K $3 »$ and «S $2 »$, via block system «O », receive compression stress facilitating additional strengthening and stabilizing of structure of cement stone and concrete on its basis. Herewith, chemical energy released during hydration of expanding additive may be effectively used for provision of prestress of tendon and forming of plane or spatial prestressing of reinforced concrete of building structures, which is practically impossible to provide by means of mechanical, thermal and thermomechanical stressing of tendons. 


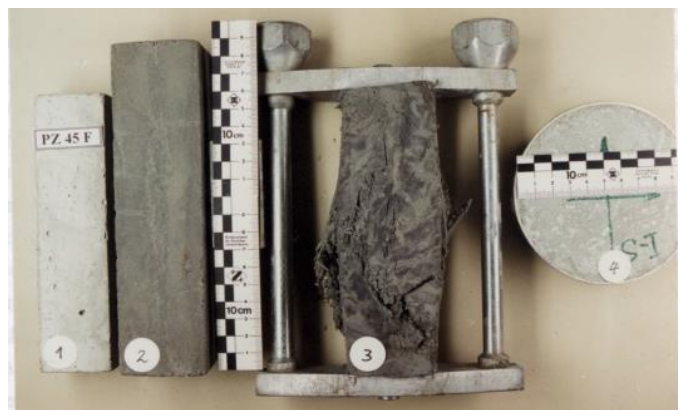

Fig. 3. The influence of the degree of restriction of expansion deformations on change in volume of expanding concrete C $30 / 37(2 \div 4)$ : 1 - fine-grained concrete without expanding additive at normal hardening; 2 - hardening with no restriction of deformations (expansion is $185 \mathrm{~mm} / \mathrm{m}$ ); 3 - singleaxis restriction of expansion (expansion pressure is $3.9 \mathrm{~N} / \mathrm{mm}^{2}$ ) $; 4$ - two-axis restriction of expansion (expansion pressure is $15.3 \mathrm{~N} / \mathrm{mm}^{2}$ )

At long-term stressed state element «P» switches to operation, which corresponds to the development of plastic deformations of cement stone and is defined with plastic yield value or with plastic strain modulus. Herewith, some relaxation of stressed state is observed that demonstrates itself in reduction of its level by $5-10 \%[8,9]$.

Experiments proved that at two-axis restriction of expansion deformations expansion in the direction perpendicular to plane amounts not more than $15 \%$ of the potential expansion value $[9,11]$. Herewith, expansion potential means maximum possible expansion without any external restrictions. Study of quality of microstructure of expanding cements hardening at different conditions of deformation restriction performed with application of optical and scanning electronic microscope demonstrated that expanding cements hardening without restriction of expansion deformations have sufficiently homogeneous porous microstructure.

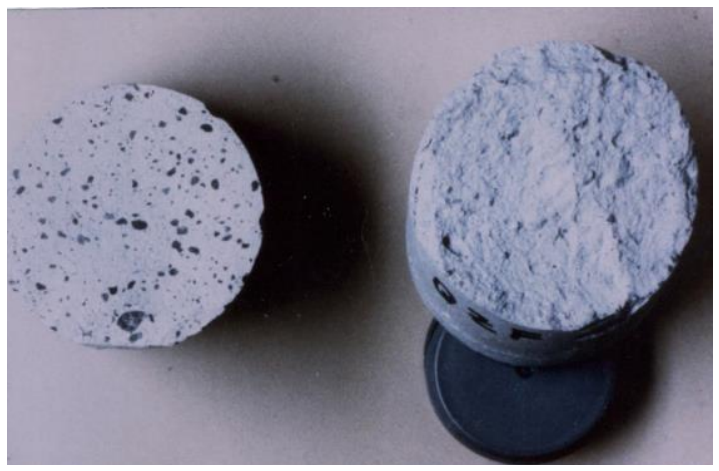

Fig. 4. Formation of black aggregates within the volume of cement stone hardening under conditions of isotropic restriction of deformations (specimen to the left) and absence of aggregates at hardening with no restriction of deformations (specimen to the right) at expansion potential of $185 \mathrm{~mm} / \mathrm{m}$.

The structure of expanding cement stone hardening at restriction of expansion deformations is substantially denser than the structure of cement stone that hardens freely $[12,13]$. However, at the analysis of microstructure and products of hydration of expanding cements hardening at complete three-axis restriction of expansion deformations an 
abnormal phenomenon was established in the form of black inclusions with the diameter of up to $5 \mathrm{~mm}$ distributed uniformly in the volume of hardened cement stone (Figure 4).

Optically it reminds the structure of concrete with coarse black filler. Further thorough analysis of microstructure of cement stone with expanding additive on sulfoaluminate basis showed that the entire volume of hardened cement stone beside coarse agglomerates contains black inclusions with the size of 5-50 $\mu \mathrm{m}$.

Analysis of microstructure of detected black inclusions, as well as their contact area with the main volume of cement stone conducted using the electronic microscope with the resolution of up to 1 x 10000 did not reveal any essential difference in their structural characteristics, i.e. the presence of any transition area. General structure is characterized with highly dense fine-pored structure free of microcracks (Figure 5).

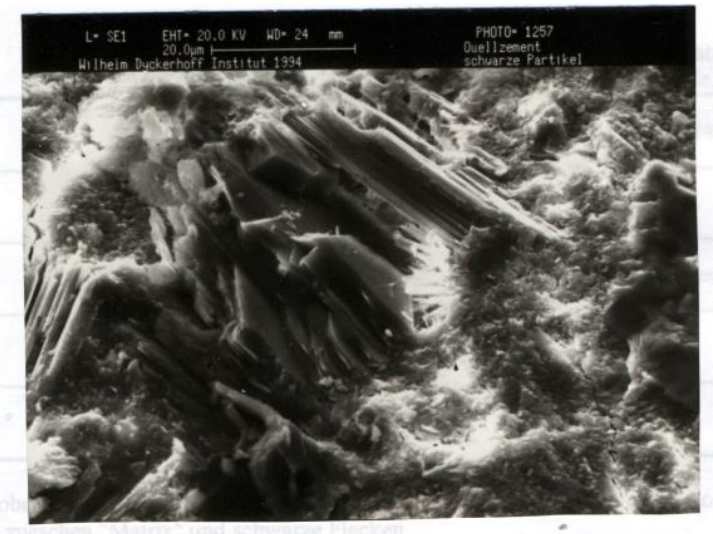

Fig. 5. Mixed crystal of hydration products (ettringite, Portlandite, hydrosilicate) within the black aggregate volume: magnification is $1 \times 10000$.

At thorough study of structure and composition of black agglomerates it is established that they are presented with mixture of hydration products consisting of mixture of hydrosilicates, Portlandite and hydrated sulfoaluminates. Herewith, the morphology of mixture of crystalline hydrates located within the volume of black inclusions is represented with "cold" alloy of mutually intergrown crystals of ettringite, monosulfate and Portland cement bound with the hydrosilicate phase. X-ray phase analysis, spectral analysis and differential thermal analysis allowed quantitative determination of phase composition of black inclusions, which is presented in Table 1.

Table 1. The results of X-ray phase analysis, differential thermal analysis and ISA of hydration products after statistical processing.

\begin{tabular}{|c|c|c|c|c|}
\hline $\begin{array}{c}\text { Hydration } \\
\text { products }\end{array}$ & Specimen 1 & Specimen 2 & Specimen 3 & Specimen 4 \\
\hline Ettringite & $36.7 \%$ & $62.3 \%$ & $57.7 \%$ & $55.8 \%$ \\
\hline Monosulfate & $22 \%$ & small amount & small amount & small amount \\
\hline Portlandite & $4 \%$ & $5,8 \%$ & $5,4 \%$ & $4,9 \%$ \\
\hline Calcite & $6.4 \%$ & $9.0 \%$ & $10.2 \%$ & $8.9 \%$ \\
\hline
\end{tabular}

Note: specimen 1 is the grey portion or matrix; specimens 2 and 4 are the black agglomerates; specimen 3 is the transition area between the "matrix" and the black agglomerates. 
On the basis of analysis of experimental results it is established that the black inclusions appear only at complete restriction of expansion deformations higher than 73 psi. With the increase of expansion pressure higher than 73 psi up to set experimental value of 754 psi the number of black inclusions increases proportionally. This phenomenon may be illustrated using the rheological model. It has already been mentioned that at increase of expansion potential under conditions of complete restriction of expansion deformations strengthening elements «Y $3 »$ and «Y4» and springs «K1» and «K2» switch on.

At further expansion structural elements «P» and «S1» experience compressive stress both from the piston «V» and from the spring «Ko», i.e. these elements are being in a compressed state, which provides their interpenetration and merging in the form of mixed crystalline hydrates. Herewith, element «P» represents hydrosilicate phase C-S-H, and element «S» represents crystalline hydrates, in the form of Portlandite $\mathrm{CH}$, ettringite C3A3C9 32H, monosulfate C3A9C9 $12 \mathrm{H}$ and calcite CaCO3.

The process of forming of mixed crystalline hydrate phase develops under conditions of excessive pressure, which influence changes solubility of initial components and equilibrium state of ions in aqueous medium, where the processes of crystalline hydrates formation develop. This affects the morphology of formed hydration products and reveals itself in the form of formed mixed crystalline hydrates and in properties of cement stone and concrete on expanding cement basis, which hardens under complete restriction of expansion deformations: increased density of cement stone structure, high strength at compression, increased crack resistance and durability.

\section{Conclusions}

The processes of forming of structure and properties of cement stone and concrete on its basis are clearly illustrated using the rheological model. Quantitative assessment of elements comprising the model creates a prerequisite for mathematic modeling of processes of structuring of cement stone and concrete accounting for "external" and "internal" influence factors.

Experiments proved that at hardening of expanding cement systems on sulfoaluminate basis at complete restriction of expansion deformations are formed "abnormal" mixed crystalline hydrates with high strength and density of structure, uniformly distributed across the volume of cement stone. The process of formation of "abnormal" crystalline hydrates is clearly illustrated using the proposed rheological model.

All tests were carried out using research equipment of The Head Regional Shared Research Facilities of the Moscow State University of Civil Engineering (RFMEFI59317X0006).

\section{References}

1. TU 21-20-18-80: Self-stressing cements. Technical specifications. Moscow, Stroyizdat, 26 (1980)

2. I. Chartschenko, J. Stark, Wiss. Zeitschr. Hochsch. Arch. BauwesenWeimar, 39/3, 163-171 (1993)

3. I. Chartschenko, Theoretische Grundlagen zur Anwendung von Quellzementen in der Baupraxis- Habilitation. BRD. Weimar, 198 (1995)

4. I. Charschenko, J. Stark, Second CANMET Symposium of Advances in Concrete Technology, 62-71 (1999)

5. I. Chartschenko, V. Rudert, H-D. Wihler, Zement-Kalk-Gips 49, 21-30 (1996)

6. Yu. A. Piskunov, G.A. Davydov, Technologies of reinforced concrete on the basis of self-stressing cements, 76 (1989) 
7. H. Tilman, VDM Verlag, Saarbrucken, 276 (2009)

8. L. Alimov, I. Kharcenko, V. Voronin, MATEC Web of Conferences 106, 02004 (2071) SPbWOSCE-201 (2016)

9. I.Ya. Kharchenko, A.P.Pustovgar, S.A.Pashkevich 10.18720|MCE.75.16 7 (75), 162172 (2017)

10. Y.M.Bagenov, I.J. Harcenko, A.K. Djatlov, 19.Internationale Baustoftagung IBAUSIL, 326-329 (2015)

11. W.A. Alekseev, A.I.Harcenko, I.J. Harcenko, 19.Internationale Baustoftagung IBAUSIL, 835-839 (2015)

12. W.A. Alekseew, S.I.Bagenova, С.И., І.J. Harcenko, WZ «Ziwilbau» 9, 33-37 (2016)

13. W.A. Alekseew, S.I. Bagenova, I.J. Harcenko, S.- E. Beterbiev, WZ "Westnik MGSU" 11, 48-59 (2016) 